\section{ZNF277 microdeletions, specific language impairment and the meiotic mismatch methylation (3M) hypothesis}

European Journal of Human Genetics (2015) 23, 1113; doi:10.1038/ejhg.2014.262; published online 24 December 2014

We were intrigued by the pedigrees in the paper by Ceroni et al ${ }^{1}$ in the October 2014 issue that explored the association between ZNF277 microdeletions and specific language disorder (SLI). The authors make the case for ZNF277 microdeletions contributing to the risk of SLI while acknowledging that there was little evidence of co-segregation of these microdeletions with SLI in their discovery pedigree plus eight other families. The authors only discuss this discrepancy in terms of reduced penetrance, that is, unaffected siblings that carry the microdeletion, but say little about the reverse discordance in two of their three multiplex families, that is, where one of the two affected siblings has inherited the maternal microdeletion and the other has not. This reverse discordance has been reported in multiplex families with autistic spectrum disorder (ASD) in which a putative risk copy number variation $(\mathrm{CNV})$ is segregating. ${ }^{2}$ This paper on ASD, and our finding of a maternal grandmother age effect in ASD prompted us to propose the meiosis mismatch methylation (3M) hypothesis. ${ }^{3}$ This states that, in female early meiosis I, the pairing of a chromosome carrying a microdeletion with a wild-type homologue increases the chance of abnormal methylation due to chromosome looping through misaligned pairing, such as would occur with silencing of a transposon. ${ }^{4}$ This in turn results in the functional silencing of the wild-type gene when transmitted to offspring. $3 \mathrm{M}$ predicts that all offspring of a woman carrying a risk CNV are at risk of the condition even though only half inherit the CNV. Independently, around the time of formulating the $3 \mathrm{M}$ hypothesis, an ASD family was reported in which one affected sibling had inherited a maternal deletion containing the oxytocin receptor gene (OXTR) and the other affected sibling had no deletion, but had epigenetic misregulation of this gene through aberrant gene silencing by DNA methylation. ${ }^{5}$ We suggest that it would be useful to examine at least the DNA methylation status of the maternally inherited ZNF277 allele in the siblings that have SLI but not the microdeletion.

\section{CONFLICT OF INTEREST}

The authors declare no conflict of interest.

Marcus Pembrey ${ }^{\star, 1,2}$, Jean Golding ${ }^{1}$ and Jessica Connelly ${ }^{3}$ ${ }^{1}$ Centre for Child and Adolescent Health, School of Social and Community Medicine, University of Bristol, Bristol, UK;
${ }^{2}$ Institute of Child Health, University College, London, UK; ${ }^{3}$ Department of Psychology, University of Virginia, Charlottesville, Virginia, USA E-mail: M.Pembrey@bristol.ac.uk

1 Ceroni F, Simpson NH, Francks C et al: Homozygous microdeletion of exon 5 in ZNF277 in a girl with specific language impairment. Eur J Hum Genet 2014; 22: 1165-1171.

2 Bucan M, Abrahams BS, Wang K et al: Genome-wide analyses of exonic copy number variants in a family-based study point to novel autism susceptibility genes. PLoS Genet 2009; 5: e1000536.

3 Golding J, Steer C, Pembrey M: Parental and Grandparental Ages in the Autistic Spectrum Disorders: A Birth Cohort Study. PLoS One 2010; 5: e9939.

4 Walsh CP, Chaillet JR, Bestor TH: Transcription of IAP endogenous retroviruses is constrained by cytosine methylation. Nat Genet 1998; 20: 116-117.

5 Gregory SG, Connelly JJ, Towers AJ et al: Genomic and epigenetic evidence for oxytocin receptor deficiency in autism. BMC Med 2009; 7: 62.

\section{Reply to Pembrey et al: 'ZNF277 microdeletions, specific language impairment and the meiotic mismatch methylation (3M) hypothesis'}

European Journal of Human Genetics (2015) 23, 1113-1115; doi:10.1038/ejhg.2014.275; published online 24 December 2014

In a recent paper, ${ }^{1}$ we described a homozygous exonic microdeletion in ZNF277 in a girl with specific language impairment (SLI). This microdeletion was also identified in the heterozygous form in eight families of the SLI Consortium (SLIC) cohort and four families with ASD cases from the IMGSAC Cohort. We observed an increased allelic frequency of ZNF277 microdeletions in SLI probands (1.1\%) compared with both ASD family members $(0.3 \%)$ and unrelated controls $(0.4 \%)$, suggesting that these microdeletions might be a risk factor for SLI. However, as the ZNF277 microdeletions showed incomplete segregation with the SLI phenotype, as they were also identified in unaffected family members and, in some cases, they were not inherited by the affected children (reverse discordance), we hypothesised that these CNVs might contribute to the SLI susceptibility in a complex manner, acting as a risk factor with a reduced penetrance.

Pembrey and colleagues have suggested that an epigenetic mechanism may account for the reverse discordance observed for the maternal ZNF277 microdeletions. According to their hypothesis, called meiotic mismatch methylation $(3 \mathrm{M}){ }^{2}$ during the maternal meiosis I, 